\title{
Jam, ou sobre pesquisa colaborativa em Arquitetura e Urbanismo
} Jam, or about collaborative research in Architecture and Urbanism

\author{
Marcelo Tramontano \\ Nomads.usp, Universidade de São Paulo, Brasil \\ tramont@sc.usp.br \\ Gabriele Landim \\ Nomads.usp, Universidade de São Paulo, Brasil \\ gabriele.landim@usp.br
}

\author{
Dyego Digiandomenico \\ Nomads.usp, Universidade de São Paulo, Brasil \\ digiandomenico@usp.br \\ Mayara Dias de Souza \\ Nomads.usp, Universidade de São Paulo, Brasil \\ dias_mayara@yahoo.com.br
}

\begin{abstract}
Within the scope of JAM research project being developed at Nomads.usp (www.nomads.usp.br), in collaboration with research groups from two other Brazilian public universities, this article focuses on the issue of communication between participants of remote collaborative design processes mediated by digital technologies to design buildings with complex shapes. The aim is to contribute towards reflecting on the theme crowdthinking exploring, on the one hand, issues related to the structuring of research projects in Architecture on the subject, and, on the other hand, aspects of remote collaborative design processes.
\end{abstract}

Keywords: Parametric design; Digital fabrication; Architectural design; Collaborative processes; BIM.

\section{Introdução}

Em desenvolvimento no Nomads.usp - Núcleo de Estudos de Habitares Interativos, ligado ao Instituto de Arquitetura e Urbanismo da Universidade de São Paulo (IAU-USP), Brasil, o Projeto Jam trata das dinâmicas de comunicação envolvidas em processos colaborativos de projeto à distância e da realização local de seus produtos. Contida em seu título está a principal hipótese da pesquisa: em tais processos, as dinâmicas de comunicação aparentam-se às da conversação musical que estruturam uma jam session. De fato, ambas lidam com as ideias de improviso e indeterminismo (Feisst, 2013), pressupondo que cada participante precisa estar atento às informações que recebe dos demais, devendo processálas rapidamente utilizando, para isso, seu próprio saber técnico, produzir novas informações que serão, por sua vez, recebidas e processadas pelos demais, e assim por diante.

\section{Formulando a conversação}

Jam é um projeto multifacetado que visa contribuir para a reflexão sobre transdisciplinaridade e colaboratividade em pesquisa, na subárea de Projeto de Arquitetura e Urbanismo. Através de estudos teóricos e experimentos práticos, a pesquisa trata de processos digitais de projeto de edificações que se utilizam de geometrias complexas, em interações remotas e presenciais, durante as fases de concepção, fabricação e montagem, com a participação de universidade, comunidade e indústria. A pesquisa pretende também favorecer a atualização tecnológica de setores produtivos locais, ao explorar a fabricação digital e montagem em escala real da edificação projetada, em cidades distintas. Seus principais objetivos são:

1. Formular processos colaborativos de projeto mediados por programas de design paramétrico, especialmente aqueles de plataforma BIM (Building Information Modelling), e por técnicas de fabricação digital para prototipagem de modelos e produção de componentes construtivos;

2. Compreender e ampliar os protocolos de troca de informação em plataforma BIM a partir de recursos disponíveis em variados aplicativos computacionais;

3. Instrumentalizar a formação de redes de colaboração para pesquisa e projeto nas áreas de arquitetura e construção;

4. Entender requisitos para a construção de parcerias com empresas locais para a produção de componentes, fornecimento de materiais e mão-de-obra especializada;

5. Debater estratégias para inserção dos processos estudados na formação de estudantes de arquitetura, engenharia e áreas relacionadas.

Projeto Jam envolve três grupos de pesquisa, de universidades públicas situadas em três Estados brasileiros diferentes, em cidades com características bastante diversas: Nomads.usp, da Universidade de São Paulo, na cidade de São Carlos, São Paulo; Nó Lab, da Universidade Federal de Viçosa, em Viçosa, Minas Gerais; e Algo+ritmo, da Universidade Federal de Mato Grosso do Sul, em Campo Grande, Mato Grosso do Sul.

As etapas de trabalho agrupam-se em quatro blocos, cada um com duração de aproximadamente nove meses: 1. preparação da infraestrutura e capacitação das equipes localmente, testes de comunicação entre as equipes, exploração de programas computacionais, sistematização dos referenciais teóricos e metodológicos; 2. elaboração à distância, pelas três equipes parceiras, de um único projeto 
arquitetônico parametrizado; 3 . desenvolvimento de projetos locais por cada equipe a partir do projeto inicial, por alterações de valores em seus parâmetros em função de interações com parceiros locais do setor produtivo e da comunidade; 4. produção e montagem de objeto arquitetônico em meio urbano, em cada uma das três cidades, ações de avaliação do projeto e do processo. Cada bloco finaliza-se com um seminário de avaliação em uma das instituições, reunindo presencialmente todos os colaboradores do bloco. Todas as atividades estão sendo registradas em vídeo, fotos e relatórios.

As atividades da pesquisa estão em curso de realização há alguns meses, iniciando um cronograma de três anos. Estão sendo usados programas como Rhinoceros e Revit, e seus editores gráficos de algoritmos Grasshopper e DynamoBIM, dentre vários outros propostos espontaneamente pelos participantes, associados ao uso de cortadoras a laser, fresadoras CNC e impressoras 3D. Além da revisão bibliográfica, de estudos exploratórios de programas computacionais e do exame de referências arquitetônicas, destaca-se, dentre as etapas já realizadas, um workshop de projeto desenvolvido ao mesmo tempo presencialmente e à distância via Internet, abordado a seguir neste artigo. O workshop envolveu pesquisadores, professores e alunos das três instituições parceiras.

Dentro da abrangência do projeto, o presente artigo concentra-se na questão da comunicação entre participantes de processos colaborativos de projeto à distância, mediada por tecnologias digitais, na concepção de edificações com formas complexas. Busca-se contribuir para a reflexão sobre o tema crowdthinking explorando, por um lado, questões pertinentes à estruturação de projetos de pesquisa em Arquitetura relacionados ao assunto, e, por outro lado, aspectos relativos a processos colaborativos de projeto à distância.

\section{Jamming together remotely}

O workshop de projeto realizado no âmbito do projeto Jam teve como principais objetivos: 1 . ampliar o entendimento dos participantes sobre o uso de dados computacionais em processos de projeto de arquitetura, 2. explorar recursos computacionais e de comunicação à distância para fomentar a colaboratividade, e 3 . esboçar conexões entre o design paramétrico e a fabricação digital dos componentes construtivos do projeto. Foram definidas atribuições distintas, mas não estanques, para professores (como organizadores, viabilizadores e observadores do conjunto da atividade), pesquisadores (acompanhamento das equipes e auxílio no uso dos scripts) e alunos de graduação (participantes das equipes de projeto). Tanto as discussões coletivas relacionadas ao projeto quanto as reuniões dos organizadores e as avaliações finais foram realizadas via Internet, através de variados meios, síncrona e assincronamente. As equipes foram formadas com, em média, dois alunos de cada instituição, compondo-se quatro equipes de cinco a sete alunos cada uma. Durante sete sessões, cada uma com aproximadamente três horas de duração, foram desenvolvidas propostas de pavilhões destinados a abrigar atividades culturais e de lazer na praça Roosevelt, em São Paulo. Esse local foi escolhido por não se situar em nenhuma das três cidades participantes, o que pressupunha que o acesso dos grupos a informações sobre o sítio também se fizesse exclusivamente via Internet. Solicitou-se aos grupos que apresentassem proposições projetuais abordando questões técnico-construtivas, com estudo de implantação no sítio e inserção na paisagem, que produzissem simulações e análises de desempenho através do uso de scripts e plug-ins diversos, e que buscassem privilegiar a comunicação entre os membros da equipe através da modelagem física e digital.

\section{Trabalho colaborativo}

Para verificar as premissas de trabalho colaborativo propostas pelo projeto Jam, era fundamental que cada equipe fosse constituída por participantes das três cidades, estimulando o debate e a formulação de propostas projetuais à distância. Essa dinâmica retoma as conclusões e os bons resultados obtidos em experiências anteriores em trabalhos do Nomads.usp (Tramontano et al., 2005; Tramontano, Santos, 2003; Kós et al., 2005). Nela, exercitou-se novamente o conceito de um atelier expandido que inclui todos os alunos, professores e monitores participantes, e cuja comunicação se dá tanto presencialmente, no âmbito local, quanto remotamente. Como já se havia verificado nas experiências anteriores, esse modelo de organização estimula relações menos hierárquicas entre participantes que não se conhecem pessoalmente, priorizando o foco nos conteúdos discutidos.

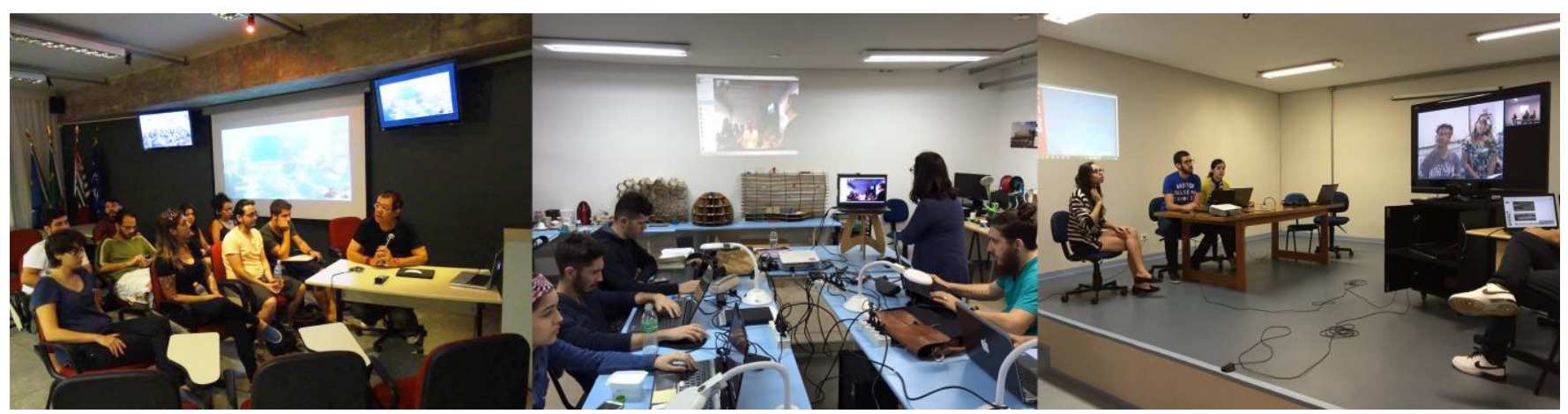

Figura 1: Equipes em desenvolvimento de projeto conectadas simultaneamente na Universidade de São Paulo, na cidade de São Carlos, São Paulo; Universidade Federal de Mato Grosso do Sul, em Campo Grande, Mato Grosso do Sul; e Universidade Federal de Viçosa, em Viçosa, Minas Gerais. Fonte: Autores. 
$\mathrm{Na}$ sessão inicial, foram discutidas referências de projeto, características e condicionantes do sítio, os principais programas computacionais a serem utilizados, relações entre alguns scripts e formas arquitetônicas, e os meios de comunicação à distância escolhidos pelos participantes para troca de informações e discussões. Como todo trabalho em equipe, houve, durante as sessões, embates pontuais e pequenos desentendimentos, baseados em suposições agravadas pela prerrogativa do trabalho à distância. Confirmou-se a hipótese de que esse tipo de dinâmica demanda uma grande abertura de espírito de cada um e confiança nos demais, o que pressupõe um perfeito entendimento da proposta de trabalho a priori, clareza no estabelecimento de regras iniciais, e comprometimento em um acordo mútuo envolvendo os termos do processo e os objetivos a serem atingidos. Esses requisitos estendem-se, obviamente - e primeiramente - aos professores e organizadores do processo.

A comunicação via Facebook foi utilizada extensivamente. Desde o início, foram criados dois grupos na rede social: um, público, para comunicação e trocas de arquivos entre todos os participantes (http://bit.ly/2a1YTij), e outro, fechado, para auxiliar a comunicação entre professores. Os direcionamentos para os trabalhos foram apresentados pelos professores e discutidos no grupo público, e incluíam desde orientações das equipes, compartilhamento de referências projetuais, soluções técnicas de projeto e de uso de scripts, até a definição de pranchas, modelos e apresentações para a avaliação final. Esse suporte possibilita que todos os participantes acompanhem, discutam e esclareçam dúvidas, mesmo que tenham estado ausentes de alguma das sessões de trabalho.

\section{Comunicação}

O sucesso do desenvolvimento de projetos à distância, nos termos propostos pelo Projeto Jam, depende diretamente da qualidade de conexão à Internet. Se, por um lado, a largura de banda de cada localidade podem ser distintas, por outro lado, a estabilidade da conexão precisa ser assegurada. $O$ projeto quis, intencionalmente, colocar em comunicação grupos sediados em cidades e instituições com redes, equipamentos e conexões muito variados, no sentido de entender os aspectos mais essenciais da comunicação à distância no processo de projeto. A transmissão síncrona de vídeo pareceu menos importante do que a transmissão de áudio, pois as imagens podem ser compartilhadas por outros meios, com bastante rapidez. A transmissão síncrona da fala revelou-se tão importante que, diante de problemas de conexão, algumas equipes utilizaram-se de ligações telefônicas para se comunicar, ou mesmo de recursos VOIP. Ficou evidente, durante todo o processo, incluindo as avaliações finais, a importância da atenção que deve ser dispensada à organização e o estabelecimento de meios de comunicação eficientes e conexão à Internet estável entre todos os envolvidos. Esses aspectos influenciam diretamente o fluxo de trabalho das equipes, o controle e versionamento dos arquivos, e podem impactar enormemente a qualidade dos produtos finais.
Durante o workshop, foram testados os comunicadores Hangout, Skype e Polycom, tendo o último apresentado os melhores resultados por possibilitar maior controle de posicionamento de câmera e telas, boa qualidade de áudio e vídeo e estabilidade de conexão ao utilizar-se de link dedicado. No entanto, espontaneamente todos os grupos utilizaram outros meios de comunicação como os comunicadores do Facebook e Whatsapp. Merece destaque o uso intenso de smartphones para a comunicação, certamente por tratar-se de um meio familiar a todos os participantes, mas especialmente aos alunos. Por conter câmeras, tela de visualização, microfone e recurso de áudio em "viva voz" em um único equipamento, e também por possibilitar a instalação de diversos aplicativos de comunicação baseados no uso da Internet, o celular revelou-se um suporte valioso durante todo o processo, em momentos de naturezas bastante distintas, para uso de alunos, professores e pesquisadores. Além dessas características, o celular baseia-se nas premissas da mobilidade e da portabilidade, que acabam por estender várias atividades de projeto para além do limite temporal das sessões de trabalho.

\section{Design paramétrico}

A primeira dificuldade para a integração das equipes foi a diferença de níveis de habilidade dos participantes no uso dos programas de modelagem paramétrica propostos, Rhinoceros e Grasshopper. As discussões em torno de questões de projeto foram às vezes dificultadas e mesmo interrompidas pela necessidade de aprendizado dos programas utilizados no workshop. Foram apresentados aos alunos scripts do Grasshopper como referência, com a intenção de estimular a exploração de geometrias menos usuais, procurando desconstruir o vínculo entre o programa e formas complexas exclusivamente curvas. Ampliar o repertório sobre materiais e sistemas construtivos capazes de viabilizar a construção de edificações com formas complexas e não priorizar formas curvas eram metas a serem atingidas nessa etapa.

O projeto a ser produzido no workshop era o de uma edificação de pequeno porte, associando o uso de programas computacionais paramétricos de projeto com saberes próprios do campo da arquitetura e do urbanismo. A intervenção arquitetônica escolhida, na praça Roosevelt, em São Paulo, consistia em uma estrutura para acolher apresentações musicais e teatrais espontâneas ou ligadas aos teatros e bares existentes no entorno. Trabalhou-se com a possibilidade de que essa estrutura pudesse promover maior integração entre os freqüentadores dos bares e teatros e a praça, transformando ou reutilizando equipamentos já existentes na praça, como a arquitetura do pergolado ou a base utilizada pela Guarda Civil Metropolitana, e considerando áreas com piso em diferentes níveis. Também esperava-se que a intervenção dialogasse com a comunidade de skatistas, bastante presente na praça. A escolha do local estimulou a definição de alguns parâmetros como desníveis, acústica, fluxos, visuais, entorno.

\section{Fabricação digital}

A produção de modelos físicos, parciais ou reduzidos, é entendida, no Projeto Jam, como parte integrante e 
indissociável do processo de projeto. No workshop, quis-se verificar a pertinência de se materializar, localmente em cada instituição, modelos das edificações concebidas colaborativamente à distância, comparando-se os resultados obtidos em relação à qualidade e acuidade dos modelos, às dificuldades de produção e à sua utilidade no processo de projeto. Foram utilizadas as tecnologias de corte a laser, impressão 3D e fresagem CNC. Cada universidade participante possuía pelo menos uma máquina com uma dessas tecnologias, e produziu, juntamente com os alunos, um vídeo tutorial sobre o seu uso, acompanhado de um passoa-passo, disponibilizados posteriormente no grupo de discussões. Todas as sessões de fabricação foram acompanhadas pelos organizadores e pesquisadores e registradas em vídeos, compartilhados em seguida no grupo online, para que os demais integrantes de cada equipe acompanhassem e entendessem o processo de produção de todos os modelos físicos de sua equipe.

Além da capacitação no uso de máquinas de fabricação digital, destacam-se, como resultados importantes, a ampliação do entendimento sobre processos digitais de projeto, a pré-visualização da produção de componentes construtivos do tipo file-to-factory (Tramontano, 2015), e a ampliação do leque de escolhas técnico-construtivas para projetos de arquitetura parametrizados utilizando fabricação digital.

\section{Jam, crowdthinking e design paramétrico}

A crescente adoção de modelagem paramétrica nas fases de concepção de projetos de arquitetura pode solicitar inicialmente maior integração da equipe, uma vez que os rápidos avanços nos paradigmas de projeto exigem dos designers o aprimoramento dos conceitos de pensamento algorítmico, formalizações da linguagem de programação e uso de programas específicos. No início do workshop, foram apresentados aos participantes os conceitos de organização em programação modular definidos por Daniel Davis, Jane e Mark Burry (2011), orientando as equipes nas ações de desenvolvimento dos algoritmos, e demonstrando a relevância de se sistematizar títulos, entradas, saídas, grupos e descrições. Estes conceitos de organização tornam o compartilhamento do código mais colaborativo por aumentar significativamente sua legibilidade.

No entanto, durante o desenvolvimento dos projetos, foram identificadas dificuldades dos alunos em compartilhar e colaborar de forma remota e organizada no desenvolvimento dos algoritmos no Grasshopper. A cada etapa de revisão dos projetos, por parte das equipes, os scripts tornavam-se menos legíveis. Verificaram-se, então, problemas de versão e compartilhamento de códigos, uma vez que o Grasshopper não possui normas bem estabelecidas para organização e estruturação dos códigos. Na prática, percebeu-se a queda de produtividade das equipes na concepção do projeto por ter que corrigir erros de código e incompatibilidade de versões. Essa condição evidenciou o potencial do BIM enquanto plataforma central de gerenciamento e modificação de projetos de maneira colaborativa, que permite a criação de modelos paramétricos associativos e o gerenciamento de suas informações considerando os diversos agentes que interagem no projeto, durante todos os ciclos e fases, da concepção à pós-ocupação. O BIM pressupõe a criação de um repositório centralizado de dados e informações, ao qual todos os agentes que interagem no projeto podem recorrer continuamente. Assim, o designer estabelece os dados apenas uma vez, permitindo a atualização de todas as informações de maneira conjunta quando alguma modificação é efetuada no modelo (Eastman et al. 2011).

O uso do BIM com programação visual transcende o entendimento de "ferramentas" de concepção de projeto, na mesma medida que exige do designer a compreensão ampliada sobre arranjos produtivos e detalhes técnicos, mudando a maneira como ele interage com outros agentes envolvidos no processo, como profissionais da engenharia e construção, usuários do futuro espaço construído e com a própria equipe nas tomadas de decisão. Embora as contribuições e avanços do BIM no desenvolvimento e integração do processo de projeto sejam relevantes, a colaboração dentro dessa plataforma está focada na edição da geometria e sua documentação através da modelagem de informação. Cabe, assim, aos pesquisadores explorar plataformas abertas que comportem o desenvolvimento colaborativo e interativo da lógica de programação e algoritmos aplicados no processo de projeto. $\mathrm{O}$ arquiteto Daniel Davis afirma que a engenharia de software é "um precedente importante para os arquitetos que utilizam modelos paramétricos", com implicações críticas de como esses modelos são utilizados na prática (Davis, 2013, p. 57, tradução nossa).

Dentro de um processo de projeto colaborativo, especialmente com agentes remotos, a organização, a clareza e o gerenciamento dos dados é fundamental na otimização da participação dos membros e da produção da equipe. Em outras áreas do conhecimento, encontramos sistemas usados amplamente na otimização do trabalho colaborativo, que podem ser referências a processos de projeto em arquitetura baseados no uso de algoritmos. Destaca-se o Sistema de Controle de Versão (Version Control System ou VCS), amplamente utilizado na Ciência da Computação e Engenharia de Software.

O Sistema de Controle de Versão documenta todas as alterações produzidas em um ou diversos arquivos, permitindo aos usuários recuperar versões específicas dos arquivos, identificar e comparar modificações realizadas de forma compartilhada, tornando possível rastrear as alterações feitas no arquivo. Sistemas de controle de versão estão divididos em centralizados e distribuídos. Nos sistemas centralizados, os usuários trabalham em cópias dos arquivos hospedadas em um único servidor central. Esse sistema é comum na plataforma BIM, que permite o trabalho colaborativo em um mesmo arquivo, mas com a centralização da permissão de alterações no projeto. Nos sistemas distribuídos, os repositórios são autônomos e independentes, um para cada colaborador, e os repositórios podem se comunicar diretamente. Trabalhar em sistemas distribuídos muda o fluxo de trabalho do projeto colaborativo, pois permite a colaboração entre diferentes grupos de pessoas, de diversas maneiras, simultaneamente no mesmo projeto. Ou seja, a contribuição no projeto pode ser feita de maneira sincronizada entre todos, sendo transmitida de um repositório para outro, sem existir hierarquia de importância entre eles.

É, portanto, de grande relevância investigar sistemas de versionamento e organização que possam ser incorporados 
no processo de projeto de arquitetura através de recursos computacionais. Essa demanda se apresenta com evidência quando se pensa em processos colaborativos que empregam a lógica da programação e algoritmos, especialmente através da programação visual com Grasshopper e DynamoBIM, que constituem um excelente meio de exploração das potencialidades da parametrização, do início da concepção à montagem final das edificações.

\section{Conclusões}

A formulação de processos colaborativos de projeto à distância deve partir da necessidade de se enriquecer a experiência projetual, otimizar a comunicação entre participantes, em todos os níveis, e compartilhar informações precisas e completas sobre os produtos que estão sendo desenvolvidos. Inversamente, no entanto, percebe-se que a prática de se escolher um programa principal como ponto de partida de tais processos implica em ter que lidar continuamente com suas funcionalidades e limitações, e resolver problemas que possivelmente não se colocariam a partir de uma atitude mais holística dos formuladores do processo.

O uso de programas e plataformas variados em um mesmo processo de trabalho colaborativo à distância em arquitetura pode permitir a inclusão no processo de grupos em situações muito distintas em termos de infraestrutura e de habilidades no uso de determinados programas. Seria essa uma tentativa de se superar diferenças grandes entre instituições de ensino e pesquisa da área, não apenas no vasto território brasileiro mas também entre países.

De fato, processos colaborativos de projeto à distância renovam compreensões locais sobre o fazer arquitetônico, estimulando a colocação conjunta de novas questões no interior mesmo do processo de projeto: por exemplo, no desenho de detalhes construtivos que consideram especificidades regionais difíceis de serem reconhecidas de outra forma. É também nesse sentido que a fabricação digital pode ser de grande ajuda. Ao trazer para o âmbito da tangência física elementos construtivos até então apenas visualizados em ambientes digitais, o modelo físico permite compreensões, alterações e ajustes que, a partir de novas digitalizações, realimentarão o processo de projeto inclusive com informações que antecipem etapas de execução na obra.

\section{Referências}

Davis, D. (2013) Modelled on Software Engineering: Flexible Parametric Models in the Practice of Architecture. PhD Dissertation, RMIT University, p. 57

Davis, D., Burry, J., \& Burry, M. (2011). Untangling Parametric Schemata: Enhancing Collaboration through Modular Programming. In CAAD Futures 2011: Designing Together, p. 5568

Eastman, C., Teicholz, P., Sacks, R., Liston, K. (2011) BIM handbook: a guide to building information modeling for owners, managers, designers, engineers, and contractors, 2nd ed. Hoboken, NJ: Wiley.

Feisst, S. (2013) Losing control: Indeterminacy and improvisation in music since 1950.1 Disponível em: <http://www.newmusicbox.org/articles/Losing-ControlIndeterminacy-and-Improvisation-in-Music-Since-1950/> Acesso em: 08 jun. 2016.

Kos, J., Malveira, T., Cabral Filho, J., Mascarenhas, E., Tramontano, M. (2005) Low-tech remote collaborative design studios. In: CAADRIA 10th International Conference on Computer Aided Architectural Design Research in Asia, 2005, New Delhi. Proceedings.

Tramontano, M., Benevente, V., Marques, S., (2005) Habitar a cidade: algumas lições de uma experiência de ensino. In: Projetar 2005 II Seminário sobre Ensino e Pesquisa em Projeto, 2005, Rio de Janeiro. Projetar 2005 II Seminário sobre Ensino e Pesquisa em Projeto. Rio de Janeiro: UFRJ.

Tramontano, M. (2015) Quando pesquisa e ensino se conectam: design paramétrico, fabricação digital e projeto de arquitetura. In: XIX Congresso da Sociedade Ibero-Americana de Gráfica Digital SIGRADI, 2015, Florianópolis. Project information for interaction. Florianópolis: UFSC. v. 2. p. 544-550.

Tramontano, M., Santos, M. (2003) International Design Studio 2002: exercício de projeto. In: Projetar 2003, 2003, Natal, RN. Anais CDRom, 2003. 